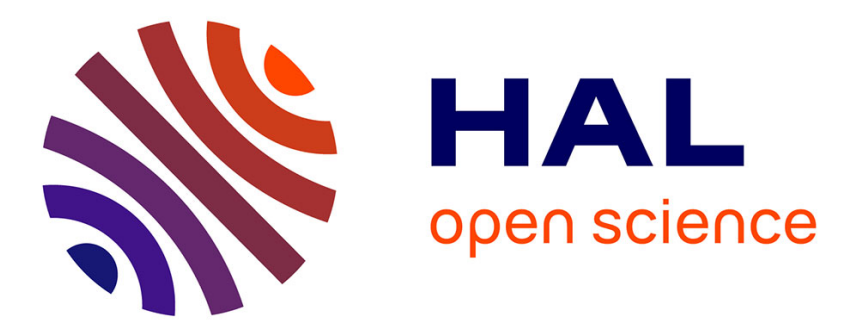

\title{
Voss surfaces: A design space for geodesic gridshells
} Nicolas Montagne, Cyril Douthe, Xavier Tellier, Corentin Fivet, Olivier Baverel

\section{To cite this version:}

Nicolas Montagne, Cyril Douthe, Xavier Tellier, Corentin Fivet, Olivier Baverel. Voss surfaces: A design space for geodesic gridshells. Symposium of the international Association for Shell and Spatial Structures, Aug 2021, Surrey, United Kingdom. hal-03364545

\section{HAL Id: hal-03364545 \\ https://hal.science/hal-03364545}

Submitted on 4 Oct 2021

HAL is a multi-disciplinary open access archive for the deposit and dissemination of scientific research documents, whether they are published or not. The documents may come from teaching and research institutions in France or abroad, or from public or private research centers.
L'archive ouverte pluridisciplinaire HAL, est destinée au dépôt et à la diffusion de documents scientifiques de niveau recherche, publiés ou non, émanant des établissements d'enseignement et de recherche français ou étrangers, des laboratoires publics ou privés. 


\title{
Voss surfaces: A design space for geodesic gridshells
}

\author{
Nicolas MONTAGNE* a,b $^{\text {a }}$ Cyril DOUTHE ${ }^{\mathrm{a}}$, Xavier TELLIER ${ }^{\mathrm{a}}$, Corentin Fivet ${ }^{\mathrm{b}}$, Olivier Baverel $^{\mathrm{a}}$ \\ a Laboratoire Navier, UMR 8205, Ecole des Ponts, IFSTTAR, CNRS, UPE \\ 77455 Champs-sur-Marne - MLV Cedex 2 - France \\ nicolas.montagne@enpc.fr
}

${ }^{\mathrm{b}}$ EPFL, ENAC, Structural Xploration Lab, Passage du Cardinal 13b CH-1700 Fribourg

\begin{abstract}
The design of envelopes with complex geometries often leads to construction challenges. To overcome these difficulties, resorting to discrete differential geometry proved successful by establishing close links between mesh properties and the existence of good fabrication, assembling and mechanical properties. In this paper, the design of a special family of structures, called geodesic shells, is addressed using Voss nets, a family of discrete surfaces. The use of discrete Voss surfaces ensures that the structure can be built from simply connected, initially straight laths, and covered with flat panels. These advantageous constructive properties arise from the existence of a conjugate network of geodesic curves on the underlying smooth surface. Here, a review of Voss nets is presented and particular attention is given to the projection of normal vectors on the unit sphere. This projection, called Gauss map, creates a dual net which unveils the remarkable characteristics of Voss nets. Then, based on the previous study, two generation methods are introduced. One enables the exploration and the deformation of Voss nets while the second provides a more direct computational technique. The application of theses methodologies is discussed alongside formal examples.
\end{abstract}

Keywords: Voss nets, gridshell, geodesics, constructive geometry, Chebyshev nets, Gauss map.

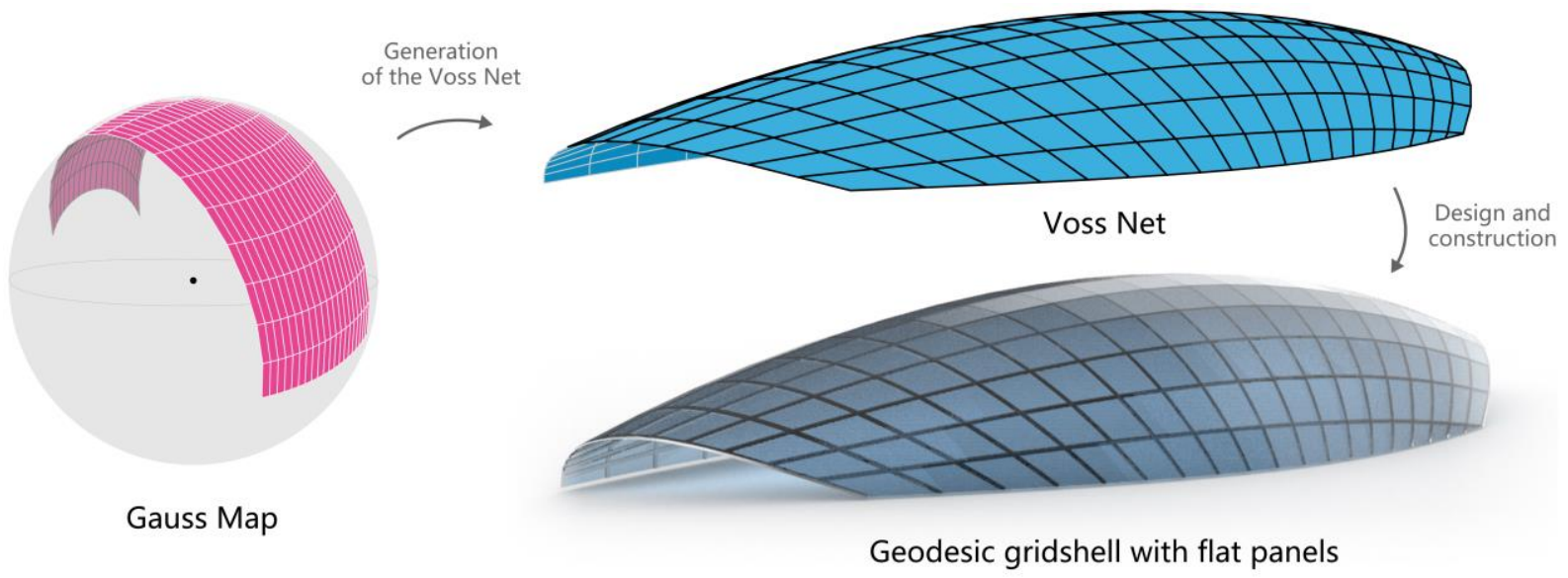

Figure 1: Overview of the design of geodesic gridshell using Voss nets

\section{Introduction}

The construction of complex shaped envelopes gives rise to great challenges in terms of manufacturing and assembling. The design of the support structure must conciliate competing requirements, ranging 
from mechanical efficiency, through architectural intents, to cost effectiveness. Two approaches coexist for the construction of complex shaped envelopes. Adopting the traditional way of building, the first one relies on stiff elements assembled with rigid connections (Schlaich and Schober [21], Mesnil et al. [8], Tellier et al. [23]) while the other strategy uses slender beams bent elastically. The later type of structure, referred to as active-bending structures (Lienhard et al. [6]), presents the benefit to smoothly approximate the target geometry. In the great majority of cases, rectangular cross section beams are applied tangent to the surface (Harris et al. [4], Liddell [5], Colabella et al. [2]). However, in such structures, the arrangement of the members often results in excessive stresses and the covering requires the use of tailor-made doubly-curved elements.

The problem of designing shells with actively bent rectangular elements was tackled by Natterer et al. [10] who made use of geodesic lines. Geodesic lines are curves on surfaces with a vanishing geodesic curvature. Hence, a lath following a geodesic line is not bent sideways, along its strong axis and avoids excessive stresses and local instabilities. Inspired by Natterer et al. [10], Pirazzi and Weinand [13] proposed a framework for the design of shells with geodesic lines, called geodesic shells, and applied it for the realization of a pavilion.

Recently, discrete differential geometry, appeared as an elegant and powerful way to address the challenges faced during the design of complex shapes. The constructive expectations are translated as geometrical constraints on the mesh, mesh which is a discrete representation of the doubly-curved envelope. Using this point of view, geodesic lines were further studied for the mapping of surfaces (Pottmann et al. [15]) or for the modelling of developable surfaces (Rabinovitch et al. [16]). In this context, Douthe et al. [3] presented a strategy for the covering of elastic gridshells with flat panels based on isoradial meshes. The covering property results from the duality of the grid structure, called a Chebyshev net, with a conjugate network of curves. However, the problem of covering geodesic shell with flat quad was not addressed in the literature.

\subsection{Related works}

In this paper, the design of geodesic shells is addressed through a special family of discrete surfaces called Voss nets. Resorting to these surfaces guarantees that the support structure can be built from initially-straight rectangular cross-section beams that are elastically bent and can be covered using only flat quadrilateral panels. Unveiled by the eponymous mathematician in 1888 in their smooth version (Voss [24]), discrete Voss surfaces, or Voss nets, are defined as quadrangular meshes in which opposite angles made by edges around a vertex are equal and where each face is planar (Sauer and Graf [18]). Voss nets have been studied since the first half of the $19^{\text {th }}$ century (Wunderlich [25], Sauer [19]) and have recently resurfaced in the contexts of origami pattern and graphic statics.

Noticing their rigid-foldability property retrieved by Schief et al. [20], Tachi [22] identified Voss nets as a generalization of the well-known eggbox origami pattern. Indeed, this pattern can be modelled by a mesh with planar faces and whose opposite angles at edges are equal. In that approach, Voss nets are discussed in a jagged version, suited for the origami application. Such description decouples Voss nets from their comprehension as discretization of smooth Voss surfaces, and is consequently unrelated with the geodesic approach developed in this paper.

Mitchell et al. [9] addressed Voss nets in the context of graphic statics, highlighting their duality with another family of nets called K-nets. K-nets are the discrete counterpart of surfaces with constant negative Gaussian curvature. They show that, using this duality, Voss nets and K-nets can be respectively interpreted as the form and the force diagrams. Accordingly, a generation method for Voss nets is presented. However, since it requires K-nets as input, which are non-trivial 3-dimensional nets, the methodology reveals laborious. The authors built an origami pavilion based on the rigid-foldability property of Voss nets. 


\subsection{Overview}

In section 2. The Gauss map, the representation of Voss nets through normal vectors is examined. This approach gives rise to inherent nets on the unit sphere, the Gauss maps, and unveils unique characteristics of Voss nets. Then, two generation methods, based on the properties identified in the previous section, are presented in section 3. Morphogenesis. The first one addresses the design of Voss nets through their normals and results in the exploration of a linear space (Figure 1). The second uses two boundary curves and permits a more direct computation of Voss nets. Finally, these methodologies are applied for the creation of discrete Voss surfaces.

\section{The Gauss map}

The Gauss Map or spherical projection, in classical differential geometry, is the application mapping every normal vectors of a surface to its corresponding point on the unit sphere $S^{2}$. As a result, the projection of all normals generates a new surface on $\mathrm{S}^{2}$, called without ambiguity the Gauss map. The new surface is unique and must be understood as a representation of the initial surface.

In discrete differential geometry, the notion of Gauss Map is not unique. Nets, or discrete surfaces, are defined by their sets of vertices, edges and faces, thus normal vectors can be defined upon any of these entities. Moreover, several definitions of normal vectors can concur on each sets. Hence, dealing with a new family of surfaces, the challenge is to find the most adapted set or sets of normals, i.e. the sets of normal vectors that represent the characteristic properties of the underlying surfaces the best.

\subsection{Face normals}

PQ NETS: Voss nets are a subset of the larger family of planar quadrilateral nets (PQ nets). In this context, the discrete Gauss map can be defined upon the faces and is the application associating each face normals of the net to a point on the unit sphere. Following this definition, the Gauss map produces a net on $\mathrm{S}^{2}$ that is topologically dual to the initial net: each face, edge, and vertex of the initial PQ net is transformed respectively to a vertex, edge or face on the unit sphere, and conversely.
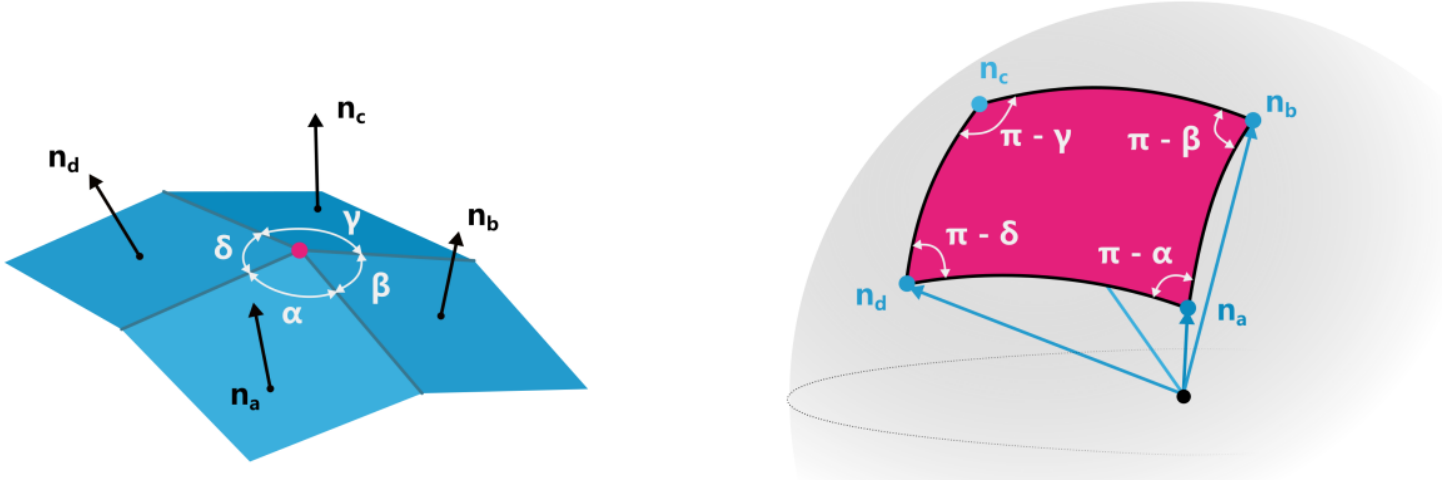

Figure 2: Spherical projection of face normals. Angles around a vertex (left) are projected on the unit sphere to supplementary angles in the dual face (right).

The projection of the face normals around a vertex leads to a useful property on the angles on the unit sphere. Let's consider the situation depicted in Figure 2, where four normal vectors form a face on the unit sphere, dual to the central vertex on the PQ net. The angles made by the edges incident to the vertex are transformed by the Gauss map to their supplementary angles in the dual face on $S^{2}$.

VOSS NETS: The above property has an immediate consequence in the special case of Voss nets. Recalling the equality of opposite angles around vertices of Voss nets, it follows that opposite angles in faces of their Gauss map are also equal. Thus, each face of the Gauss map is a spherical parallelogram, which by definition means that the Gauss map of a Voss net is a Chebyshev net of $S^{2}$ (Pinkall [12]). 

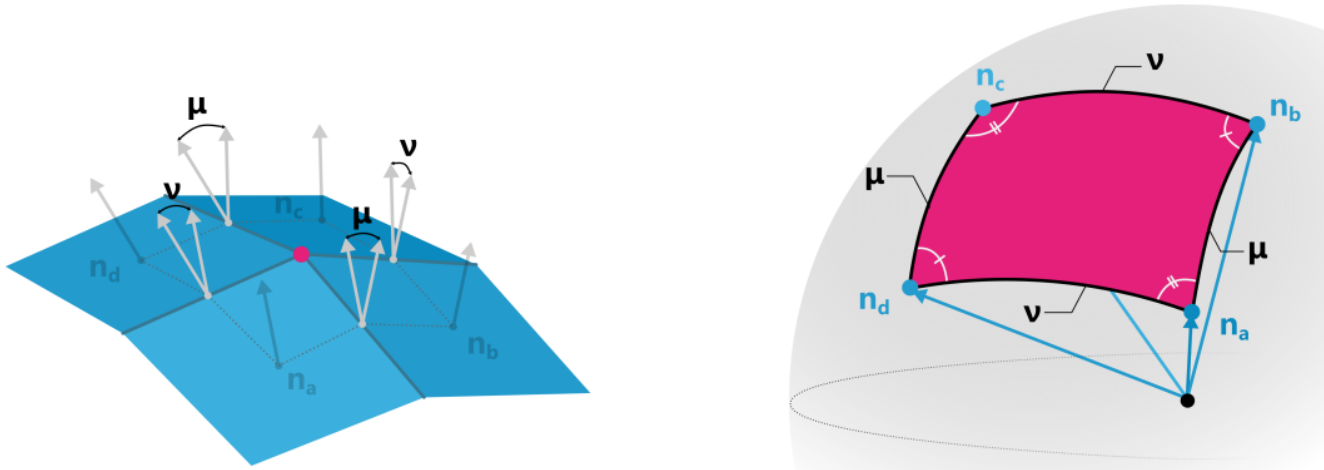

Figure 3: Spherical projection of dihedral angles of a Voss net. The angles made by adjacent face normals (left) are equal to the arc length of the edge in the Gauss map connecting these normals (right).

The Gauss map, defined upon the face normals of a PQ net, finds another interpretation with regards to dihedral angles. Dihedral angles, or angles made by the normal vectors of two adjacent faces, can be identified as the arc length of the edge which links the normal of these two faces in the Gauss map (Figure 3). For Voss nets, the faces of the Gauss map form parallelograms. Thus, each quad face of the Gauss map has equal opposite angles, which translate to the fact that these faces have equal opposite edge lengths. Therefore, following Figure 3, opposite dihedral angles around a vertex are equal. Extending this remark to the whole net, dihedral angles are constant along a coordinate lines in Voss nets. According to Schief et al. [20], this property of constancy constitutes an alternative definition for Voss nets: they are PQ nets whose Gauss map forms a Chebyshev net on $\mathrm{S}^{2}$.

\subsection{Vertex normals}

Another possibility to define a Gauss map for Voss nets consists in describing it upon the vertices. This approach is based on the study of curves and more particularly on the definition of tangent and normal vectors. Considering $C$ a smooth regular curve, non-degenerate at arc length $s$, i.e. $C^{\prime}(s)$ and $C^{\prime \prime}(s)$ are not null, then the Frenet frame of $C$ at $s$ is defined as the orthonormal frame $F_{s}^{C}=\left(t_{s}, n_{s}, b_{s}\right)$, where $t_{s}$ is the tangent vector, $n_{s}$ the normal vector and $b_{s}$ the binormal vector. A discrete counterpart of the Frenet frame can defined upon the vertices of polylines. With $P$ a polyline, non-degenerated at the vertex $p_{i}$, i.e. $p_{i-1}, p_{i}$, and $p_{i+1}$ are not aligned, the Frenet frame $F_{i}^{P}=\left(t_{i}, n_{i}, b_{i}\right)$ at $p_{i}$ is given by:

$$
t_{i}=\frac{\delta p_{\bar{i}}-\delta p_{i}}{\left\|\delta p_{\bar{l}}-\delta p_{i}\right\|}, \quad n_{i}=\frac{\delta p_{\bar{l}}+\delta p_{i}}{\left\|\delta p_{\bar{l}}+\delta p_{i}\right\|}, \quad b_{i}=t_{i} \wedge n_{i} .
$$
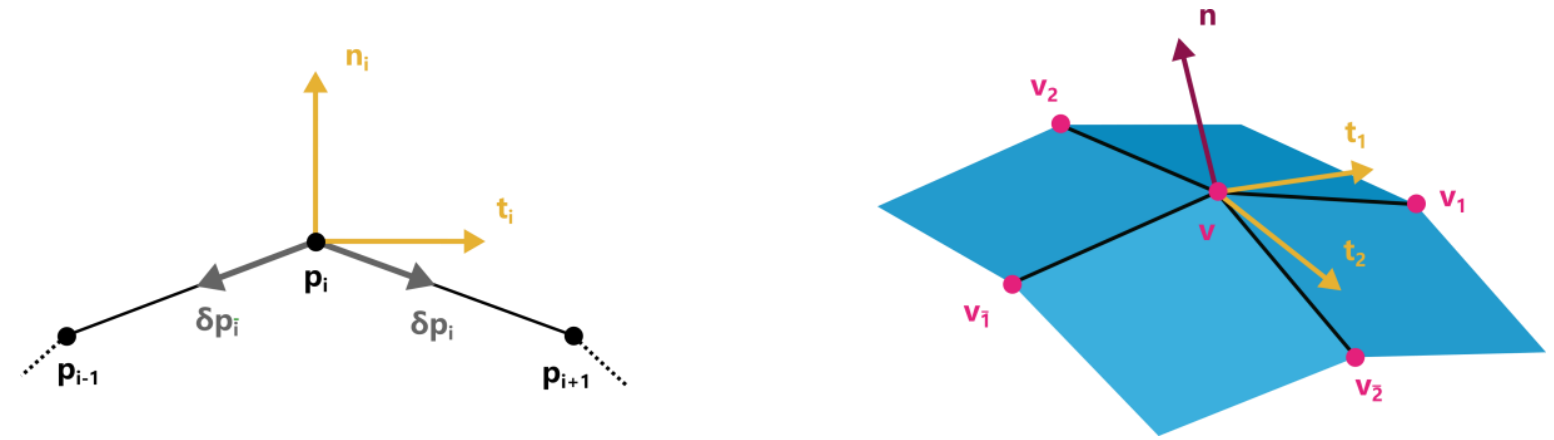

Figure 4: Frenet frame of a discrete curve (left) and vertex normal vector of a quad net (right).

QUAD NETS: Quadrilateral nets have the particularity to define a two-way grid of polylines, which is the equivalent of parameter-lines on continuous surfaces. Hence, two Frenet frames can be computed, 
one in each directions (see Figure 4). Following the analogy with the smooth setting, the normal at a vertex can be obtained from the tangent vectors of the Frenet frames at this point, $t_{1}$ and $t_{2}$, by,:

$$
n=\frac{t_{1} \times t_{2}}{\left\|t_{1} \times t_{2}\right\|}
$$

GEODESIC QUAD NETS: In the special case of geodesic quadrilateral nets, quad nets with equal opposite angles at each vertex, Rabinovitch et al. [16] proved that the normal $n$ (described in equation (2)) is aligned with the two normals $n_{1}$ and $n_{2}$ of the Frenet frames (Figure 5). This result mimics the behaviour of smooth geodesic lines whose normal vector always agrees with the surface's normal. Furthermore, it gives an alternative way to compute the vertex normal $n$ using one Frenet frame only.

VOSS NETS: In the case of Voss nets, the Gauss map defined from the projection of the vertex normals finds an interesting interpretation with regards to the other Gauss map defined upon the face normals. Indeed, the spherical projection of the normal vectors of four faces around a vertex produces a spherical parallelogram on the unit sphere, dual to the common central vertex on the Voss net (Figure 5). The normal of the common vertex is projected on the unit sphere to the centre of the parallelogram. The centre of the parallelogram is identified either as the centre or the intersection of the diagonals, or as the centre or the intersection of the medians.
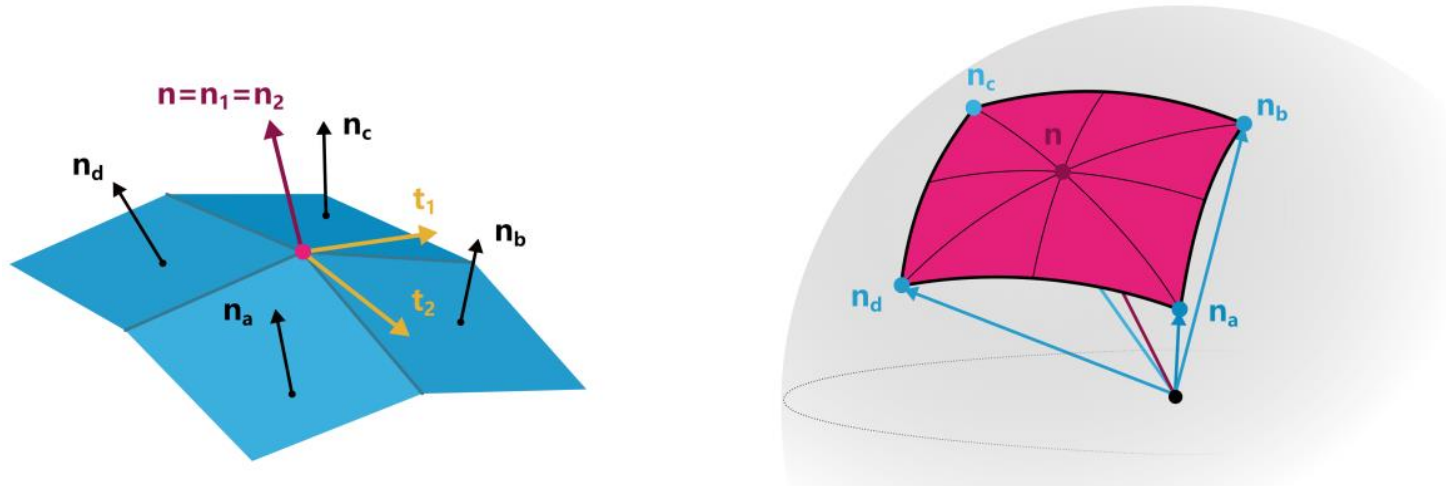

Figure 5: Spherical projection of the vertex normal vector of a Voss net.

\subsection{Choice of Gauss Map}

The above sections presented two definitions of the discrete Gauss map of Voss nets, one based on face normals, the other one based on vertex normals. The interaction between the two notions proves the pertinence of these definitions. Indeed, the superimposition of the discrete Gauss maps enriches significantly the representation of Voss nets on the unit sphere since it encompasses information on the net for both the vertices and the faces. The concomitance of the notions of Gauss maps will be advantageously exploited for the generation of Voss nets in section 3. Morphogenesis.

In the remainder, the Gauss map of Voss nets will only refer to the spherical projection of face normals since it produces a Chebyshev nets on the unit sphere, in the similar way as smooth Voss surfaces.

\section{Morphogenesis}

Following the study of Voss nets, and the presentation of their projection properties, this section addresses the design of this family of nets. Two significantly different generation processes are presented. The first methodology consists in the exploration of a linear space of Voss nets. Starting from the Gauss map, it enables the creation and deformation of Voss nets within the same framework. Then a more straightforward methodology is exposed, in which Voss nets are generated from two boundary curves. 


\subsection{Shape space exploration}

The presented methodology starts from the generation of a Chebyshev net on the unit sphere, and explores an underlying linear space composed of parallel Voss nets. Indeed, different Voss nets can be projected on a same Gauss Map, and if so, they have parallel edges.

Voss nets have the particularity to be projected through the Gauss map onto Chebyshev nets. Chebyshev nets are a well-known family of discrete surfaces and their generation has been broadly studied (Otto $e t$ al. [11], Popov et al. [14], Bouhaya et al. [1], Sageman-Furnas et al. [17]). In Masson et al. [7], different strategies are exposed for the computation of Chebyshev nets on surfaces starting either from two boundary curves (primal conditions: Figure 6a) or based on the main diagonal and the next diagonal line (dual conditions: Figure $6 \mathrm{~b}$ ). Each time the generation of the net relies on the length equality of opposite edge in each quad face. In addition, Masson et al. [7] also studied the use of mixed conditions, the introduction of singularity, and the applicability of patches of Chebyshev nets on general surfaces (Figure 6). The method exposed allows to take advantage of the various possibilities for the generation of Voss nets.
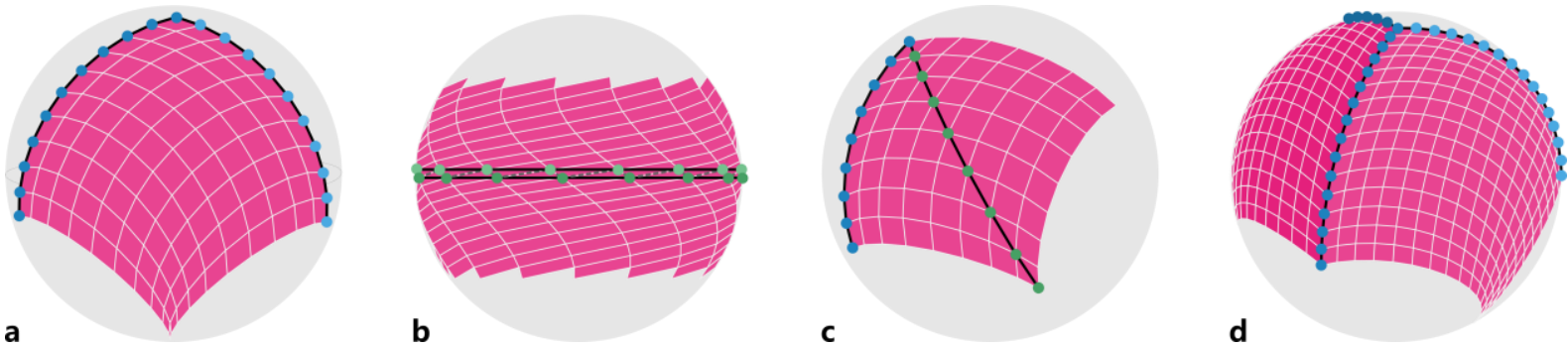

Figure 6: Generation methods for Chebyshev nets : Primal (a), dual (b), mixed (c) and patch (d) conditions.

Then, from the Chebyshev net on $\mathrm{S}^{2}$ interpreted as the Gauss Map of Voss nets, the direction of the edges of the underlying Voss nets can be retrieved. The edges common to two adjacent faces are parallel to the cross product of the normal vectors of these faces. The orientation of those normal vectors are defined by the position of the vertices in the Chebyshev net. As a consequence, all the possible Voss nets projecting on a same Gauss map have parallel corresponding edges. In fact, they form a linear space which only depends on the initially generated Chebyshev net on the unit sphere. Each vector of this linear space represent a Voss net.
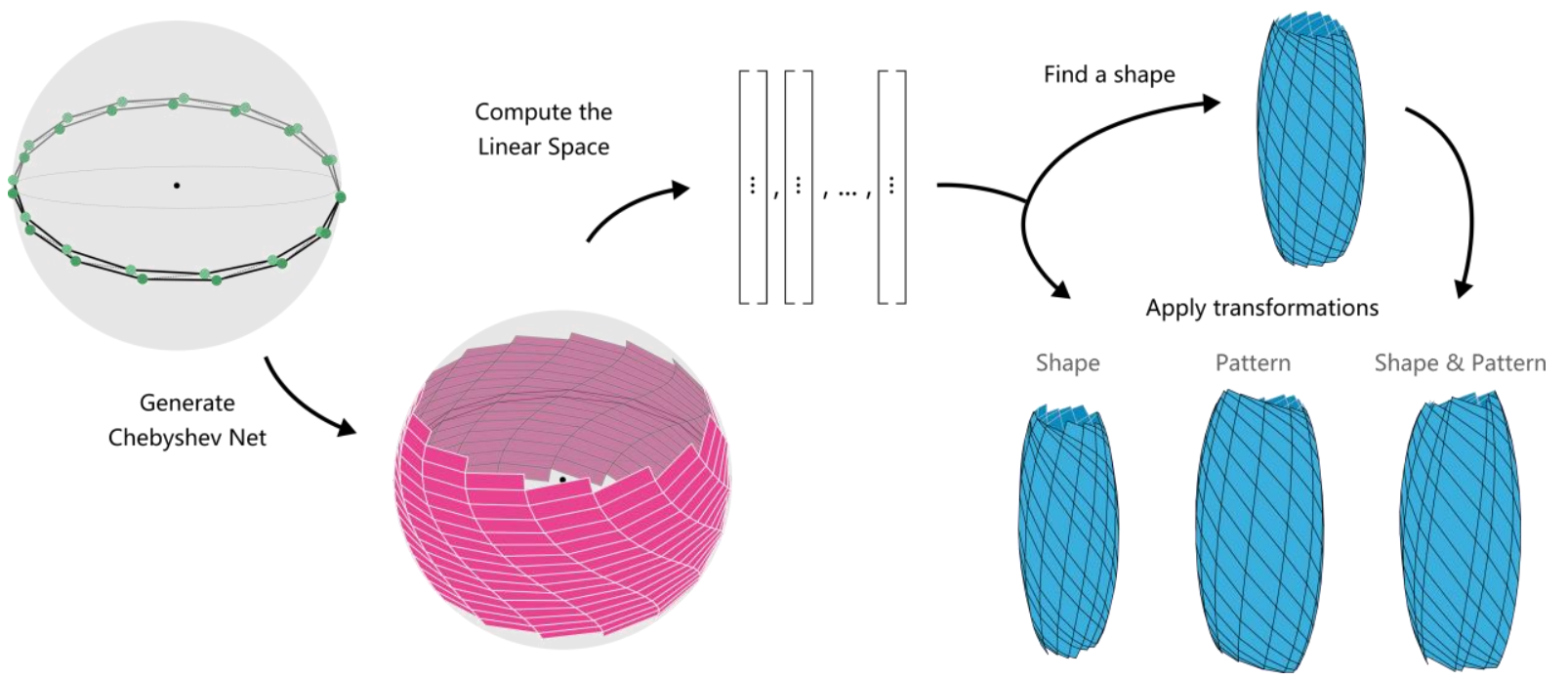

Figure 7: Summary of the exploratory generation process of Voss nets. 
Thus, using an adapted basis, the linear space of parallel Voss nets can be explored to find a suitable geometry. Since all Voss nets belong to a same linear space, several nets can be added to one another and linear combinations can be formulated in order to blend different shapes together. Thus, from this point of view, each vector of the linear space is a Voss net but also correspond to a deformation mode if it is added to another element (Figure 7).

\subsection{Explicit generation}

In this section an alternative generation process is presented. Unlike the previous methodology which addresses the morphogenesis from an exploratory point of view, this method uses two curves in space and produces the single corresponding Voss net.

Let's consider two non-degenerate polylines in space starting at the same point which represent the boundaries of the Voss net to generate. Since the input curves are not degenerate, the Frenet frames can be computed. Understanding those curves as future geodesic lines on a Voss surfaces, the normal vector in the Frenet frame gives the normal at the vertices. In addition, the first edges of each polyline define the plane of the first corner face. The normal vector of this first face is given by the cross product of theses edges.

From the projection on $S^{2}$ of the vertex normals of the boundary curves as well as the first face normal, primal conditions for Chebyshev nets can be retrieve on the unit sphere. Therefore, the specification of two boundary curves of a Voss surface is sufficient to find and generate its Gauss map.

In a similar fashion as in the previous method, the direction of the edges of the Voss net can be computed from the Gauss map. Here, in contrast with the previous method, the resolution is made locally for each face and not on the whole mesh. The prescription of the boundary curves gives the possibility to compute the faces iteratively. Starting from the first corner face, the input curves provides with the knowledge of two edge lengths $l_{1}$ and $l_{4}$ in the quad (Figure 8). Therefore, since the direction of all edges are given by the Gauss map, the face is uniquely determined and the two lengths left $l_{2}$ and $l_{3}$ are given by:

$$
\left(\begin{array}{c}
l_{1}+l_{4} \cdot \cos (\alpha) \\
l_{4} \sin (\alpha)
\end{array}\right)=\left(\begin{array}{cc}
\cos (\beta) & \cos (\alpha-\delta) \\
-\sin (\beta) & \sin (\alpha-\delta)
\end{array}\right)\left(\begin{array}{l}
l_{2} \\
l_{3}
\end{array}\right)
$$

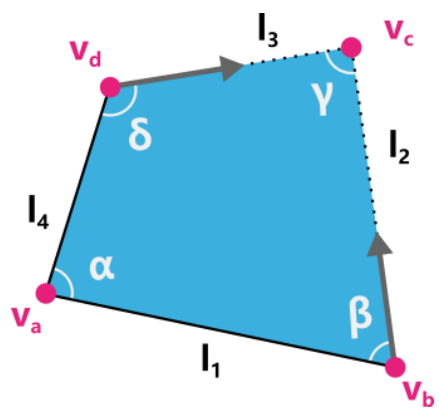

Figure 8: Configuration for the determination of the position of $v_{c}$ using equation (3)

Using the successive computation of faces, the Voss net is entirely determined. This methodology, inspired by the primal condition for Chebyshev nets and summarized in Figure 9, is permitted by the thorough knowledge of the properties of Voss nets, and particularly by the use of the interaction between the complementary notions of Gauss map upon the faces and the vertices.

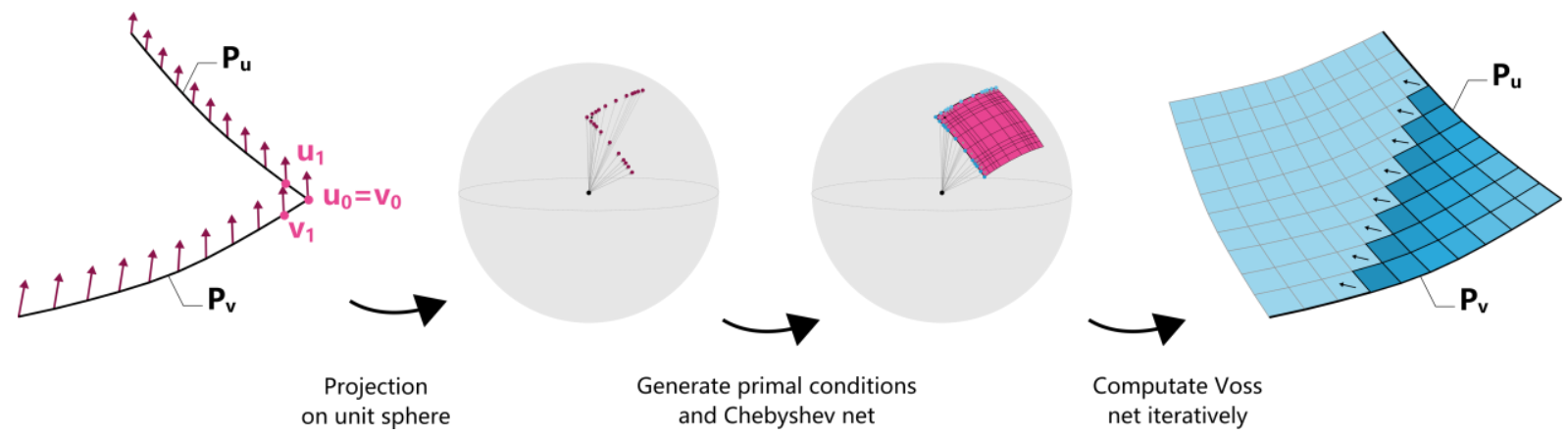

Figure 9: Summary of the explicit generation process of Voss nets. 


\subsection{Formal review}

The methodologies proposed for the creation of Voss nets allow to access various shape with positive, negative and sign-changing Gaussian curvature. The two proposed generation methods essentially differ by their design purpose.
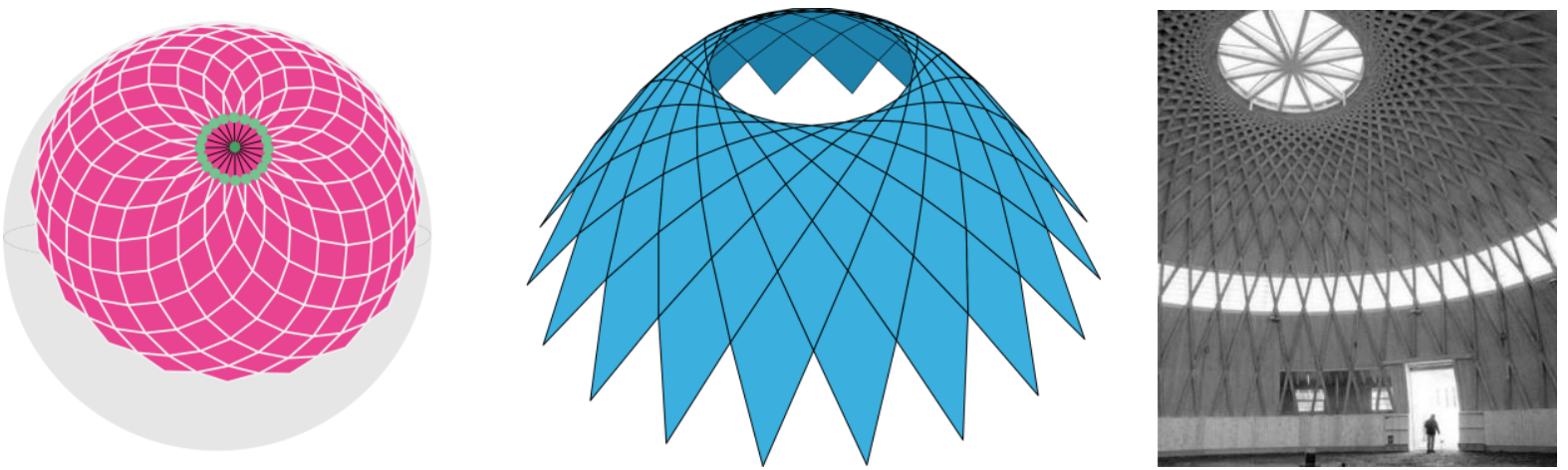

Figure 10: Voss net (centre) generated from a rosette Chebyshev net on the unit sphere (left), inspired from the geodesic shell of the equine therapy centre of Uzwil (Switzerland) by J.Natterer et al. [10] (right) [reproduction authorization pending]

On the one hand, the first exploratory method starts from an abstract design space, dual to Voss nets, which is the family of Chebyshev nets on the unit sphere. No constraint is directly expressed on the Voss nets in Euclidean space. With a certain intuition, this approach reveals particularly interesting for the design of surfaces for it notably allows the use of all the expertise acquired on Chebyshev nets (Figure 10). In addition, the manipulation of the linear space, as opposed to a single net, gives the possibility to simply transform and interpolate Voss nets.

On the other hand, the second generation process is more simple and intuitive. The Voss net is specified using explicit boundary constraints in Euclidean space. This method enables to simply patch Voss nets together in a continuous way (Figure 11).
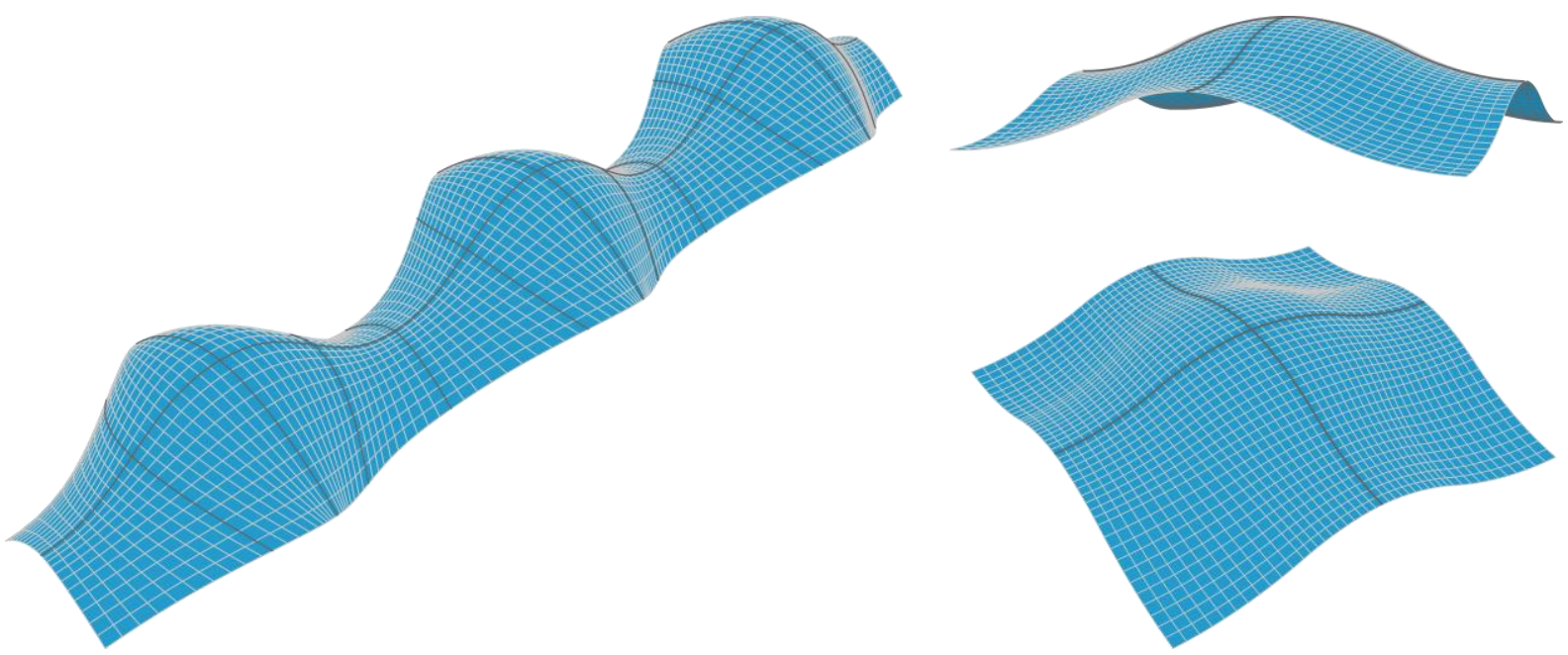

Figure 11: Voss nets created from two boundary curves. Left: Geodesic gridshell inspired by the gridshell of Downland; Right: Geodesic gridshell canopy which can be repeated in both directions. 


\section{Conclusion}

Voss nets constitute a proficient family of surfaces for the conception of doubly-curved structures. In this paper, they have been addressed for their capacity to model surfaces which can be built from elastically bent rectangular laths and covered with flat panels. In order to fully understand Voss nets, a thorough study is presented, which analyses normals vectors and their projection on the unit sphere. In particular, a rich interaction is identified between the Gauss map defined on the face normals and the one determined upon the vertices. This special characterization of Voss net is then used for the formulation of generation methods. Two methodologies are described, one permits the exploration and deformation of Voss nets while the other provides with a more explicit way to create them. Finally, the formal richness of this family is illustrated, thus supporting the relevance of the generation methods.

Further developments include the generation of the best-fit discrete surface to a target mesh. The fitting should be addressed from the Gauss map since it reduces the number of degrees of freedom for the optimisation process. In addition, further studies on transformations should identify a special conformal transformation which preserves the angle around a vertex, while deforming the net. This is supported by the fact that Voss surfaces are stable under conformal transformation in the smooth setting.

\section{Acknowledgements}

The authors warmly thank Romain Mesnil for the fruitful discussions on topics ranging from architectural geometry, to structural morphogenesis to implementation details, as well as Laurent Hauswirth for the enlightening advices in differential geometry. The authors also thank Arthur Lebée for valuable enlightenments in the domain of origami structures.

\section{References}

[1] Bouhaya L., Baverel O., and Caron J.F., Optimization of gridshell bar orientation using a simplified genetic approach, Structural and Multidisciplinary Optimization, 2014; 50 (5); 839-848.

[2] Colabella S., D'Amico B., Hoxha E. and Fivet C., Structural Design with Reclaimed Materials: An Elastic Gridshell out of Skis, in IASS 2017: Interfaces: Architecture, Engineering, Science, 2017.

[3] Douthe C., Mesnil R., Orts H. and Baverel O., Isoradial meshes: Covering elastic gridshells with planar facets, Automation in Construction, 2017; 83; 222-236.

[4] Harris R., Romer J., Kelly O. and Johnson S., Design and construction of the Downland Gridshell, Building Research \& Information, 2003; 31 (6); 427-454.

[5] Liddell I., Frei Otto and the development of gridshells, Case Studies in Structural Engineering, $2015 ; 4 ; 39-49$.

[6] Lienhard J., Alpermann H., Gengnagel C. and Knippers J., Active Bending, a Review on Structures where Bending is Used as a Self-Formation Process, International Journal of Space Structures, 2013; 28 (3-4); 187-196;

[7] Masson Y., Existence and construction of Chebyshev nets and application to gridshells, Université Paris-Est, Champs-sur-Marne, 2017.

[8] Mesnil R., Douthe C. and Baverel O., Non-standard patterns for gridshells: fabrication and structural optimization, Journal of the International Association for Shell and Spatial Structures; 2017; 58 (4); 277-286.

[9] Mitchell T., Mazurek A., Hartz C., Miki M., and Baker W., Structural Applications of the Graphic Statics and StaticKinematic Dualities: Rigid Origami, Self-Centering Cable Nets, and Linkage Meshes, in IASS 2018: Creativity in Structural Design, 2018.

[10] Natterer J., Burger N., and Müller A., The roof structure "Expodach" at the World Exhibition Hanover 2000, in Space Structures 5, 2002; 185-193.

[11] Otto F., Hennicke J., and Matsushita K., IL10 Gitterschalen. 1974. 
[12] Pinkall U., Designing Cylinders with Constant Negative Curvature, in Discrete Differential Geometry, Eds. Birkhäuser Basel, 2008, 55-66.

[13] Pirazzi C. and Weinand Y., Geodesic Lines on Free-Form Surfaces: Optimized Grids for Timber Rib Shells, in World Conference on Timber Engineering, 2006; 7; 595-601.

[14] Popov E., Geometric Approach to Chebyshev Net Generation Along an Arbitrary Surface Represented by NURBS', in International Conference Graphicon, 2002.

[15] Pottmann H., Huang Q., Deng B., Schiftner A., Kilian M., Guibas L. and Wallner J., Geodesic Patterns, ACM Trans. Graph., 2010; 29 (4).

[16] Rabinovich M., Hoffmann T. and Sorkine-Hornung O., Discrete Geodesic Nets for Modeling Developable Surfaces, ACM Trans. Graph., 2018; 37 (2).

[17] Sageman-Furnas A., Chern A., Ben-Chen M., and Vaxman A., Chebyshev Nets from Commuting PolyVector Fields, ACM Trans. Graph., 2019; 38, (6).

[18] Sauer R. and Graf H., Über Flächenverbiegung in Analogie zur Verknickung offener Facettenflache, Mathematische Annalen, 1931; 105; 499-535.

[19] Sauer R., Differenzengeometrie, Springer Verlag, 1970.

[20] Schief W., Bobenko A. and Hoffmann T., On the Integrability of Infinitesimal and Finite Deformations of Polyhedral Surfaces, in Discrete Differential Geometry, Eds. Birkhäuser Basel, 2008, 67-93.

[21] Schlaich J. and Schober H., Glass Roof for the Hippo House at the Berlin Zoo, Structural Engineering International, 1997; 7 (4); 252-254.

[22] Tachi T., Freeform Rigid-Foldable Structure using Bidirectionally Flat-Foldable Planar Quadrilateral Mesh, in Advances in Architectural Geometry, 2010, 87-102

[23] Tellier X., Douthe C., Hauswirth L. and Baverel O., Surfaces with planar curvature lines: Discretization, generation and application to the rationalization of curved architectural envelopes, Automation in Construction, 2019; 106;

[24] Voss A., Ueber diejenigen Flächen, auf denen zwei Schaaren geodätischer Linien ein conjugirtes System bilden, Math.-Naturwiss., 1888.

[25] Wunderlich W., Zur Differenzemgeometrie der Flächen konstanter negativer Krümmung, 1951 39-77. 